\title{
A Discrete Retarded Gronwall-Bellman Type Inequality and its Applications to Difference Equations
}

\author{
Feichao Chen ${ }^{1}$, Desheng $\mathrm{Li}^{1}$, and Zhang Guang ${ }^{2}$ \\ ${ }^{1}$ Tianjin University \\ ${ }^{2}$ Tianjin University of Commerce
}

February 7, 2022

\begin{abstract}
In this paper we present a new discrete retarded Gronwall-Bellman type inequality. As applications, the dynamics of some delay difference equations are studied. First, the asymptotic behavior of solutions for scalar difference equation $\$ \backslash$ Delta $\mathrm{x}(\mathrm{n})=-$ $\mathrm{a}(\mathrm{n}) \mathrm{x}(\mathrm{n})+\mathrm{B}\left(\mathrm{n}, \mathrm{x} \_\mathrm{n}\right) \$$ is discussed, and some new criterion on the asymptotic stability of the zero solution are obtained under weaker assumptions. Then the dissipativity of a nonautonomous delay difference system with superlinear nonlinearities is investigated. By using the inequalities established here, it is shown that the discrete set-valued process generated by the system possesses a unique global pullback attractor.
\end{abstract}

\section{Hosted file}

2022-Chen-Li-Zhang.pdf available at https://authorea.com/users/459225/articles/555550-adiscrete-retarded-gronwall-bellman-type-inequality-and-its-applications-to-differenceequations 